\title{
BMJ Open Does perturbation-based balance training prevent falls among individuals with chronic stroke? A randomised controlled trial
}

\author{
Avril Mansfield, ${ }^{1,2,3}$ Anthony Aqui, ${ }^{1}$ Cynthia J Danells, ${ }^{1,3}$ Svetlana Knorr, ${ }^{1}$ \\ Andrew Centen, ${ }^{2}$ Vincent G DePaul, ${ }^{4}$ Alison Schinkel-Ivy, ${ }^{5}$ Dina Brooks, ${ }^{1,3}$ \\ Elizabeth L Inness, ${ }^{1,3}$ George Mochizuki ${ }^{1,2,3}$
}

To cite: Mansfield A, Aqui A, Danells CJ, et al. Does perturbation-based balance training prevent falls among individuals with chronic stroke? A randomised controlled trial. BMJ Open 2018:8:e21510. doi:10.1136/ bmjopen-2018-021510

- Prepublication history and additional material for this paper are available online. To view these files, please visit the journal online (http://dx.doi org/10.1136/bmjopen-2018021510).

Received 2 February 2018 Revised 6 June 2018 Accepted 17 July 2018
Check for updates

(C) Author(s) (or their employer(s)) 2018. Re-use permitted under CC BY-NC. No commercial re-use. See rights and permissions. Published by BMJ.

For numbered affiliations see end of article.

Correspondence to

Dr Avril Mansfield;

avril.mansfield@uhn.ca

\section{ABSTRACT}

Objectives No intervention has been shown to prevent falls poststroke. We aimed to determine if perturbationbased balance training (PBT) can reduce falls in daily life among individuals with chronic stroke.

Design Assessor-blinded randomised controlled trial. Setting Two academic hospitals in an urban area. Interventions Participants were allocated using stratified blocked randomisation to either 'traditional' balance training (control) or PBT. PBT focused on improving responses to instability, whereas traditional balance training focused on maintaining stability during functional tasks. Training sessions were 1 hour twice/week for 6 weeks. Participants were also invited to complete 2 'booster' training sessions during the follow-up.

Participants Eighty-eight participants with chronic stroke (>6 months poststroke) were recruited and randomly allocated one of the two interventions. Five participants withdrew; 42 (control) and 41 (PBT group) were included in the analysis.

Primary and secondary outcome measures The primary outcome was rate of falls in the 12 months post-training. Negative binomial regression was used to compare fall rates between groups. Secondary outcomes were measures of balance, mobility, balance confidence, physical activity and social integration.

Results PBT participants reported 53 falls ( 1.45 falls/ person-year) and control participants reported 64 falls (1.72 falls/person-year; rate ratio: 0.85(0.42 to 1.69); $\mathrm{p}=0.63)$. Per-protocol analysis included $32 \mathrm{PBT}$ and 34 control participants who completed at least 10/12 initial training sessions and 1 booster session. Within this subset, PBT participants reported 32 falls (1.07 falls/person-year) and control participants reported 57 falls ( 1.75 falls/ person-year; rate ratio: $0.62(0.29$ to 1.30$) ; p=0.20)$. PBT participants had greater improvement in reactive balance control than the control group, and these improvements were sustained 12 months post-training. There were no intervention-related serious adverse effects.

Conclusions The results are inconclusive. PBT may help to prevent falls in daily life poststroke, but ongoing training may be required to maintain the benefits.

Trial registration number ISRCTN05434601; Results.
Strengths and limitations of this study

- This study employed an assessor-blinded randomised controlled trial. As is typical of exercise studies, participant blinding was not possible.

- Attendance to the intervention was high (mean 87\% of sessions attended), and rates of withdrawal from the study were low $(<6 \%)$.

- The primary outcome (falls in daily life) was collected via self-report, which may have led to under-reporting.

- Inclusion and exclusion criteria were minimal so that results would be generalisable to a broad population of individuals with chronic stroke. However, recruited participants were, on average, high functioning; these results might not apply to more severely affected individuals with stroke.

\section{INTRODUCTION}

People with stroke have increased fall risk compared with age-matched individuals who have not had a stroke. ${ }^{1}$ Impaired balance control, low balance confidence and high rate of falls poststroke are associated with reduced quality of life and reduced physical activity as a strategy to prevent falls. ${ }^{23}$ Physical exercise, particularly exercise that includes balance training, can reduce fall rates in older adults. ${ }^{4}$ However, studies including individuals with stroke have not demonstrated reduced fall rates following balance training. ${ }^{5} 6$

Balance training programmes typically include exercises that aim to improve the ability to maintain balance when keeping still (eg, standing with reduced base of support) or during voluntary movement (eg, sit-tostand or step ups). ${ }^{7-11}$ This type of balance training may prevent falls by reducing the risk of losing balance in daily life. However, occasional loss of balance may be an inevitable consequence of mobility, so the ability 
to react quickly after losing balance (ie, reactive balance control) is essential to prevent falls. ${ }^{12}$ Perturbation-based balance training (PBT) is a type of exercise where participants repeatedly experience loss of balance in order to practice and improve control of balance reactions. ${ }^{13}$ A review of small-sample randomised controlled trials suggests that PBT can prevent falls in older adults and individuals with Parkinson's disease. ${ }^{14}$

People with stroke have impaired reactive balance control, ${ }^{1516}$ and impaired control of balance reactions is related to increased fall rates in daily life poststroke. ${ }^{17} 18$ PBT can improve reactive balance control poststroke. ${ }^{19} \mathrm{~A}$ non-randomised study found that those who completed PBT during inpatient stroke rehabilitation fell less frequently postdischarge than those who did not. ${ }^{20}$

The main purpose of this study was to determine if PBT reduces fall rates in people with chronic stroke. A secondary purpose was to determine the effect of PBT on balance control, balance confidence, mobility, daily physical activity and social integration. We hypothesised that, compared with a control group who completed 'traditional' balance training, those who completed PBT would experience fewer falls in the year post-training and would have greater improvements in measures of functional balance and mobility. Additionally, we expected that, due to reduced fall rates and improved balance confidence, participants who completed PBT would be less likely to restrict daily physical activities; therefore, we hypothesised that participants who completed PBT would show increased daily physical activity and improved social integration compared with those in the control group.

\section{METHODS}

\section{Trial design}

This assessor-blinded pragmatic randomised controlled trial took place at the Toronto Rehabilitation Institute (University Health Network) and Sunnybrook Health Sciences Centre. Individuals with chronic stroke were recruited and randomly assigned to either: (1) PBT or (2) 'traditional' balance training (control group). The full study protocol is available elsewhere ${ }^{21}$; protocol modifications are detailed in the relevant sections below. This manuscript was prepared following the Consolidated Standards of Reporting Trials ${ }^{22}$ and Template for Intervention Description and Replication ${ }^{23}$ checklists.

\section{Participants}

Community-dwelling adults with chronic stroke $(>6$ months poststroke) were recruited from research volunteer databases and advertisements in the community. Participants could stand independently without upper limb support for $>30 \mathrm{~s}$ and tolerate at least 10 postural perturbations. Exclusion criteria were: $>2.1 \mathrm{~m}$ tall and/or weighing > $150 \mathrm{~kg}$; other neurological conditions; lower extremity amputation; unable to understand instructions in English; recent (last 6 months) significant illness, injury or surgery; severe osteoporosis (diagnosis of osteoporosis with fracture); poorly controlled diabetes or hypertension; contraindications to physical exercise ${ }^{24}$; receiving physiotherapy or supervised exercise targeting balance and mobility between the time of recruiting and the post-training assessment; and/or received PBT in the year before enrolment. Due to difficulty recruiting, the protocol was amended to allow individuals $<50$ years old to participate. Volunteers completed telephone screening and subsequently attended an initial assessment where written informed consent was obtained and eligibility was confirmed. To help alleviate barriers to participation, participants were compensated for travel expenses (public transit fare or parking).

\section{Interventions}

Participants completed two 1-hour training sessions per week for 6 weeks and two 1-hour 'booster' training sessions 3 months and 9 months after the initial training period. Interventions were administered by a physiotherapist (CJD or SK) on a 1:1 basis (ie, one physiotherapist per participant) in research laboratories in academic hospitals. Both laboratories contained a $2.63 \times 2.63 \mathrm{~m}$ 4-post XY patient lift gantry (Prism Medical, Concord, Ontario, Canada), and the Sunnybrook laboratory also contained a $8.5 \mathrm{~m}$ long ceiling lift track, to which the safety harness was attached during PBT. Physiotherapists were trained in delivering the control intervention by reviewing the intervention developers' documentation ${ }^{25}$ and in delivering the PBT intervention by study investigators (AM and VGD). Interventions followed a general guide but were tailored to participants' ability and balance impairments. Participants rated perceived level of challenge on a five-point scale (see online supplementary material) after completing each exercise set. The physiotherapists documented activities in each session, perceived level of challenge, adverse events and deviations from prescribed activities.

\section{Control group}

The control group completed the Keep Moving with Stroke programme. ${ }^{25}$ This is an exercise programme for community-dwelling individuals with stroke, based on balance and mobility interventions evaluated in clinical trials. ${ }^{9-11}$ This programme was designed to be delivered in a group but was delivered 1:1 in this study to match attention received from the physiotherapist by the PBT group. Each session included a 5-10 min warm-up, 40 min of mobility and balance exercises and a $5-10 \mathrm{~min}$ cool-down with stretching. Exercises included walking, sit-to-stand, heel raises, walking while carrying an object, tap-ups or step-ups (forward and sideways), reaching and weight shifting and standing with reduced base of support.

\section{PBT group}

PBT sessions included a 5-10 min warm-up, voluntary tasks intended to induce internal perturbations, voluntary tasks combined with external perturbations and a 5-10 min cool-down. Participants were supervised by the 
physiotherapist and wore a custom safety harness (ABG Concept Médical, Valcourt, Quebec, Canada) attached to the overhead support. Internal perturbations occurred when participants failed to control balance during voluntary movement; 'agility' tasks, such as kicking a soccer ball, were used to induce internal perturbations. External perturbations were caused by forces outside participants' control (eg, push or pull from the physiotherapist). We aimed for at least 60 postural perturbations per session and set the task difficulty such that participants required an upper extremity response, external assistance (ie, from the overhead harness or physiotherapist) or a multistep response $\sim 50 \%$ of the time. The progression in voluntary tasks occurred on a continuum from stable to mobile and from predictable to unpredictable. ${ }^{26}$ Additionally, progression occurred by increasing the magnitude of external perturbation, or imposing sensory or environmental challenges. The full PBT programme is available in the online supplementary material.

\section{Group allocation}

Participants were assigned using blocked stratified randomisation with allocation concealment to either the control or PBT group by the principal investigator (AM) who was not involved in recruiting, assessments or intervention administration. A variable block size of 4, 6 or 8 was used. There were four strata from two stratification factors: site (two levels) and frequency of 'failures' during baseline reactive balance control assessment ${ }^{17}$ (two levels). The random allocation sequence was computer generated and maintained in an electronic file by the principal investigator.

\section{Outcomes}

\section{Cohort descriptors}

Demographic and stroke information were recorded at study enrolment: age, sex, time since stroke, lesion location, falls history, National Institutes of Health Stroke Scale (NIH-SS ${ }^{27}$ and Chedoke-McMaster Stroke Assessment (CMSA) foot and leg scores. ${ }^{28}$ Demographics and medical history were obtained by self-report and, when possible, verified from participants' hospital charts.

\section{Primary outcome: falls}

A fall was defined as 'an event that results in a person coming to rest unintentionally on the ground or other lower level'. ${ }^{29}$ Participants completed 12 months of falls reporting after the initial 6-week training period. Participants were provided stamped addressed postcards containing a 2-week calendar to record falls, which they completed daily and returned to the research team fortnightly. If a postcard was not returned within 2 weeks, the research assistant called the participant to ascertain if they fell. Participants who fell completed a short telephone questionnaire regarding the cause, circumstances and consequences of the fall. Falls were excluded from analysis, by unanimous decision of two blinded research assistants, if they were caused by loss of consciousness or an overwhelming external force (ie, if anyone would fall in that situation). If the research assistants could not agree that a fall should be excluded, then that fall was included in the analysis.

\section{Secondary outcomes}

Balance and mobility and balance confidence were assessed immediately before, immediately after and 6 months and 12 months after the end of the initial training period. Functional balance and mobility were assessed using the Berg balance scale (BBS), ${ }^{30}$ the mini-Balance Evaluation Systems test (mini-BEST) ${ }^{31}$ and the Timed Up \& Go (TUG) ${ }^{32}$ The subscales of the mini-BEST were used to assess different components of balance control (anticipatory balance control, reactive balance control, sensory orientation and gait). The Activities-specific Balance Confidence (ABC) questionnaire ${ }^{33}$ was used to assess balance confidence in daily activities.

Physical activity and social integration were evaluated with the Physical Activity Scale for Individuals with Physical Disabilities (PASIPD) ${ }^{34}$ and the Subjective Index of Physical and Social Outcome (SIPSO), ${ }^{35}$ respectively, at baseline and every 2 months during the 12-month follow-up.

\section{Blinding}

The research assistants (AA and AC) were blinded to group allocation and were responsible for screening, recruiting and collecting data. At the post-training, 6-month and 12-month assessments, the research assistants guessed group allocation for each participant, rated their confidence in their guess of group allocation and noted if they had received any information to violate blinding. In cases where blinding was violated, the balance measures were recoded from video footage by another blinded research assistant.

\section{Sample size}

The target sample size was estimated for the primary outcome (fall rate in the year post-training) using a formula for negative binomial regression. ${ }^{36}$ Assuming the control group would report 1.75 per person-year, ${ }^{17} \mathrm{a}$ rate ratio of $0.54,{ }^{14}$ mean follow-up time of 11 months per person, level of significance of 0.05 and power of 0.8 , we estimated that 37 participants per group would be required to show a statistically significant between-group difference in fall rates.

\section{Statistical analysis}

Wilcoxon-Mann-Whitney test (continuous/ordinal variables) and Fisher's exact test (categorical/frequency variables) were used to compare the two groups at baseline. Negative binomial regression was used to compare fall rates, and logistic regression was used to compare the proportion of fallers between the two groups. Intent-totreat analysis was used; that is, all participants with some falls monitoring data were included in the analyses. To account for variable falls monitoring duration between participants (eg, due to premature withdrawal from the 
study), the natural log of the monitoring duration was included as an offset variable in negative binomial regression and as a covariate in logistic regression. Exploratory per-protocol analysis was also conducted, comparing proportion of fallers and fall rates between the two groups, including only those participants who attended at least 10/12 of the initial training sessions and one booster session. We initially planned to conduct repeated-measures analysis of variance, with group-by-time interaction, to evaluate the effect of the interventions on secondary outcome measures. ${ }^{21}$ However, because the variables were not normally distributed, we conducted analysis of covariance (ANCOVA), comparing BBS, mini-BEST, mini-BEST subscale scores, TUG, ABC, PASIPD, and SIPSO at each time point between groups, controlling for the value at baseline. Dependent variables were rank transformed prior to entry into the ANCOVA to allow for non-parametric analysis. ${ }^{37}$ Alpha was 0.05 for all analyses.

\section{Patient and public involvement}

Patients or the general public were not involved in the design of this study, development of research questions or outcome measures. Some participants were recruited via referral from other participants. Participants received a letter of appreciation at the end of the study, which included a summary of the results. At the end of their involvement with the study, participants were invited to complete a short questionnaire about their experiences, including whether they found data collection and the intervention difficult.

\section{RESULTS}

\section{Recruitment}

Recruiting occurred between 24 April 2014 and 29 June 2016. Initially, we planned to recruit 46 participants per group to account for a $20 \%$ withdrawal rate. ${ }^{21}$ However, recruiting was stopped when we had at least 37 participants per group who had returned at least one fall-reporting postcard. Any participants who had started the intervention at this point continued with the study until they either withdrew or completed all study elements. The trial ended when data collection for all recruited participants was complete (August 2017). Forty-four participants were assigned to each group, with 42 (control) and 41 (PBT) returning at least one fall-reporting postcard (figure 1); thus, 42 control and 41 PBT participants were included in analysis of the primary outcome (falls in daily life). Baseline characteristics for these participants are in table 1; there were no significant differences between groups on any baseline characteristics.

\section{Intervention adherence}

During the initial 6-week training programme, PBT participants attended a mean 10.5 sessions, with $34 / 41$ participants attending at least 10 sessions (out of the prescribed 12). Participants experienced a mean of 577 perturbations during all sessions (standard deviation: 195 perturbations; minimum: 42 perturbations) or a mean of 55 perturbations per session (standard deviation: nine perturbations). For all PBT sessions combined, mean rate of balance recovery 'failures' was $57 \%$, and mean rate of perceived challenge was 2.4 (on a five-point scale). In the initial 6-week training phase, control participants attended a mean of 11 sessions, with $38 / 42$ participants attending at least 10 sessions (out of the prescribed 12). On average, control participants completed $87 \%$ of the prescribed exercises (standard deviation: 18\%). For all control training sessions combined, mean rate of perceived challenge was 2.4.

\section{Outcomes and estimation}

Blinding

Blinding was violated for nine participants (seven PBT and two control participants), who revealed their group allocation in conversation with the research assistant. The BBS and mini-BEST scores for these participants were recoded from video recordings by another blinded research assistant who had no interaction with participants. For the remaining participants, the research assistants correctly guessed group allocation $56 \%$ of the time; that is, guesses were not correct more often than would be expected by random chance.

\section{Missing data}

Data were missing at assessment time points because participants: declined to complete the assessment (15 PBT and 21 control assessments); were unavailable due to acute illness (three control assessments); were unavailable due to vacation or other personal commitments (three control assessments); or could not be contacted at the time of the assessment (six control assessments). Some participants declined to come to the laboratories for the 6-month and 12-month assessments but were willing to complete the questionnaires (ABC, SIPSO and PASIPD) over the telephone. Even when participants attended a study appointment, some declined to complete individual tests; the number of individuals included in analysis of each variable at each time point is detailed in the tables.

\section{Falls}

Data on number of individuals reporting one or more falls, and fall rates, are presented in table 2. In intentto-treat analysis, the between-group differences in odds of being a 'faller' (OR: $0.71(0.30$ to 1.70$) ; \mathrm{p}=0.44)$ and fall rates (rate ratio: 0.85 ( 0.42 to 1.69$) ; \mathrm{p}=0.63$ ) were not statistically significant. Thirty-two PBT participants and 34 control participants completed at least 10/12 of the initial training sessions and one booster session and were included in per-protocol analysis. Within this subset, the between group differences in odds of being a 'faller' (OR: 0.56 (0.21 to 1.50$) ; \mathrm{p}=0.25)$ and fall rates (rate ratio: 0.62 (0.29 to 1.30$) ; \mathrm{p}=0.20$ ) were not statistically significant. 


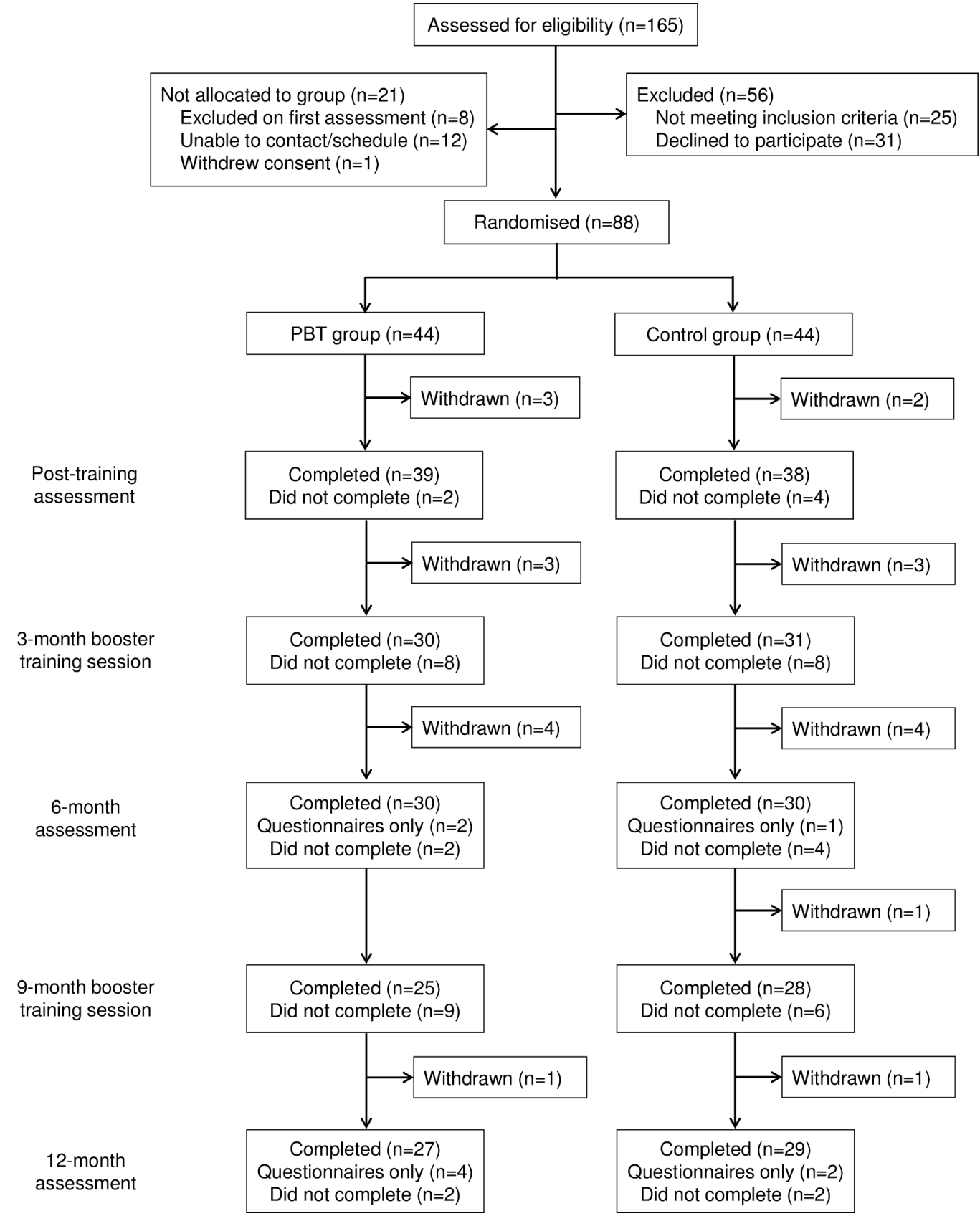

Figure 1 Participant flow through the study. Eight participants who consented to participate in the study were excluded on the initial assessment because they could not tolerate the lean-and-release postural perturbations. Participants were withdrawn after randomisation because it became apparent that they did not meet the study criteria (one PBT participant had osteoporosis with history of fracture and one control participant had uncontrolled hypertension), or because they had a significant decline in health during the training portion of the study (one PBT and one control participant). One PBT participant withdrew from the study because she did not like the group allocation. Therefore, there were 42 control participants and 41 PBT participants available for analysis of the primary outcome (falls in daily life). Participants withdrew during the 12-month follow-up period because they: no longer wished to be in the study (two PBT and one control participant); experienced a serious adverse event (two PBT, five control participants); were lost to follow-up (two PBT, three control participants); or enrolled in a conflicting study (two PBT participants). PBT, perturbation-based balance training.

Balance confidence, balance, mobility, physical activity and social integration

Post-training, the PBT group had higher scores than the control group for the reactive subscale of the mini-BEST $\left(\mathrm{F}_{174}=7.33, \mathrm{p}=0.0084\right.$; table 3$)$, whereas the control group had higher scores than the PBT group for the sensory subscale $\left(\mathrm{F}_{174}=4.19, \mathrm{p}=0.044\right)$. Scores for the reactive subscale of the mini-BEST were higher for the PBT group than the control group at 6 months $\left(\mathrm{F}_{1.57}=8.32, \mathrm{p}=0.0055\right)$ and 12 months $\left(\mathrm{F}_{1,53}=11.59, \mathrm{p}=0.0013\right)$. Likewise, at 12 months, the PBT group had a higher score on the total mini-BEST than the control group $\left(\mathrm{F}_{1,53}=4.04, \mathrm{p}=0.049\right)$. 
Table 1 Participant characteristics at study enrolment

\begin{tabular}{|c|c|c|c|}
\hline & PBT & Control & \\
\hline & $(n=41)$ & $(n=42)$ & $P$ values \\
\hline Age (years) & $66(17)$ & 67 (13) & 0.84 \\
\hline \multicolumn{4}{|l|}{ Sex (number, \%) } \\
\hline Female & $15(36.6)$ & $12(28.6)$ & 0.49 \\
\hline Male & $26(63.4)$ & $30(71.4)$ & \\
\hline $\begin{array}{l}\text { Time poststroke } \\
\text { (years) }\end{array}$ & $2.0(3.3)$ & $3.2(4.5)$ & 0.086 \\
\hline
\end{tabular}

More affected side (number, \%)

\begin{tabular}{llll}
\multicolumn{1}{c}{ Left } & $22(53.7)$ & $22(52.4)$ & $>0.99$ \\
\hline \multicolumn{1}{c}{ Right } & $19(46.3)$ & $20(47.6)$ & \\
NIH-SS (score) & $3(4)$ & $3(5)$ & 0.57 \\
\hline CMSA leg (score) & $5(1)$ & $5(1)$ & 0.54 \\
\hline CMSA foot (score) & $5(3)$ & $5(1)$ & 0.45 \\
\hline ABC scale (\%) & $65.6(26.3)$ & $79.1(33.8)$ & 0.42 \\
\hline BBS (score) & $50(10)$ & $51(7)$ & 0.94 \\
\hline Mini-BEST (score) & $18(7)$ & $18(5)$ & 0.95 \\
\hline TUG (s) & $14.4(12.3)$ & $13.0(7.6)$ & 0.62 \\
\hline PASIPD (score) & $8.4(9.5)$ & $11.6(10.9)$ & 0.48 \\
\hline SIPSO (score) & $30(9)$ & $31(13)$ & 0.74
\end{tabular}

Fall in the past year (number, \%)

\begin{tabular}{llll} 
Yes & $17(41.5)$ & $18(42.9)$ & $>0.99$ \\
No & $24(58.5)$ & $24(57.1)$ & \\
\hline
\end{tabular}

Values presented are medians with interquartile range in parentheses (for continuous/ordinal variables) or number with percentage in parentheses (for count/frequency variables). The $p$ value is for the Wilcoxon-Mann-Whitney test (continuous/ordinal variables) or Fisher's exact test (count/frequency variables). ABC, Activities specific Balance Confidence scale; BBS, Berg Balance Scale; CMSA, Chedoke McMaster Stroke Assessment; mini-BEST, mini Balance Evaluation Systems Test; $\mathrm{NIH}-$

SS, National Institutes of Health Stroke Scale; PASIPD, Physical Activity Scale for Individuals with Physical Disabilities; SIPSO, Subjective Index of Physical and Social Outcome; TUG, Timed Up \& Go.

There were no other statistically significant betweengroup differences for balance and mobility measures at any time point.

There were no significant between-group differences for the PASIPD at any time point (table 4). SIPSO scores were significantly higher for the control group compared with the PBT group at 6 months $\left(\mathrm{F}_{1,59}=6.73, \mathrm{p}=0.012\right)$, 8 months $\quad\left(\mathrm{F}_{1,54}=4.25, \mathrm{p}=0.044\right), 10$ months $\quad\left(\mathrm{F}_{1,61}=4.89\right.$, $\mathrm{p}=0.031)$ and 12 months $\left(\mathrm{F}_{1,59}=4.13, \mathrm{p}=0.047\right)$.

Data showing change in secondary outcomes over time are presented in the supplementary data (online supplementary tables S1 and S2). No analyses were conducted on these data.

\section{Ancillary analysis}

Additional exploratory analysis compared causes, circumstances and consequences of falls in daily life between
Table 2 Falls between groups

PBT Control P values

\begin{tabular}{lccc}
\hline Intent-to-treat analysis & & & \\
\hline Participants (number) & 41 & 42 & \\
$\begin{array}{l}\text { Participants reporting } \geq 1 \text { fall } \\
\text { (number) }\end{array}$ & 19 & 23 & 0.44 \\
$\begin{array}{l}\text { Falls (total number) } \\
\text { Falls (number per person- } \\
\text { year) }\end{array}$ & 53 & 64 & \\
\hline
\end{tabular}

Per-protocol analysis

\begin{tabular}{|c|c|c|c|}
\hline Participants (number) & 32 & 34 & \\
\hline $\begin{array}{l}\text { Participants reporting } \geq 1 \text { fall } \\
\text { (number) }\end{array}$ & 14 & 20 & 0.25 \\
\hline Falls (total number) & 32 & 57 & \\
\hline $\begin{array}{l}\text { Falls (number per person- } \\
\text { year) }\end{array}$ & 1.07 & 1.75 & 0.20 \\
\hline
\end{tabular}

Values presented are absolute number of participants or rate of falls per person-year. The $p$ value is for the difference in falls or fall rates from logistic regression or negative binomial regression, respectively.

PBT, perturbation-based balance training.

groups (table 5). There was a significant between-group difference in motor activity at the time of the fall $(\mathrm{p}=0.010)$. Falls in control participants were more likely to occur during transfers than falls in PBT participants, whereas falls in PBT participants were more likely to occur during reaching/bending than falls in control participants. Participants had something in their hands at the time of $45 \%$ of control group falls, compared with $23 \%$ of PBT group falls $(\mathrm{p}=0.023)$. PBT participants attempted to stop themselves from falling by using a step response for $21 \%$ or a grasping response for $18 \%$ of falls, whereas control participants tried to prevent the fall by stepping for only $9 \%$ of falls and grasping for $30 \%$ of falls; however, this difference was not statistically significant $(\mathrm{p}=0.18)$. PBT participants required assistance to get up after $48 \%$ of falls, compared with just $27 \%$ of falls for control participants $(\mathrm{p}=0.040)$. Injuries resulted from 18 falls ( $39 \%$ of falls) in the PBT group and 20 falls ( $34 \%$ of falls) in the control group ( $\mathrm{p}=0.68)$. Most injuries were minor (eg, cuts and bruises). Participants sought medical attention after three falls (all control): visit to emergency room (two falls), and treatment from an unspecified healthcare professional (one fall).

\section{Harms}

Forty-eight adverse events were possibly, probably or definitely related to study procedures or interventions among the 88 randomised participants. Events were: fatigue with training (three PBT and one control participant); joint pain during or soon after training (14 PBT 11 control participants); delayed onset muscle soreness (five PBT and eight control participants); seizure during training (one PBT participant, with history of frequent seizures); and abnormally elevated heart rate and low blood pressure during training (one control; this participant was withdrawn from 
Table 3 Balance and mobility measures between groups

$\begin{array}{lll}\text { PBT Control } & \text { P values }\end{array}$

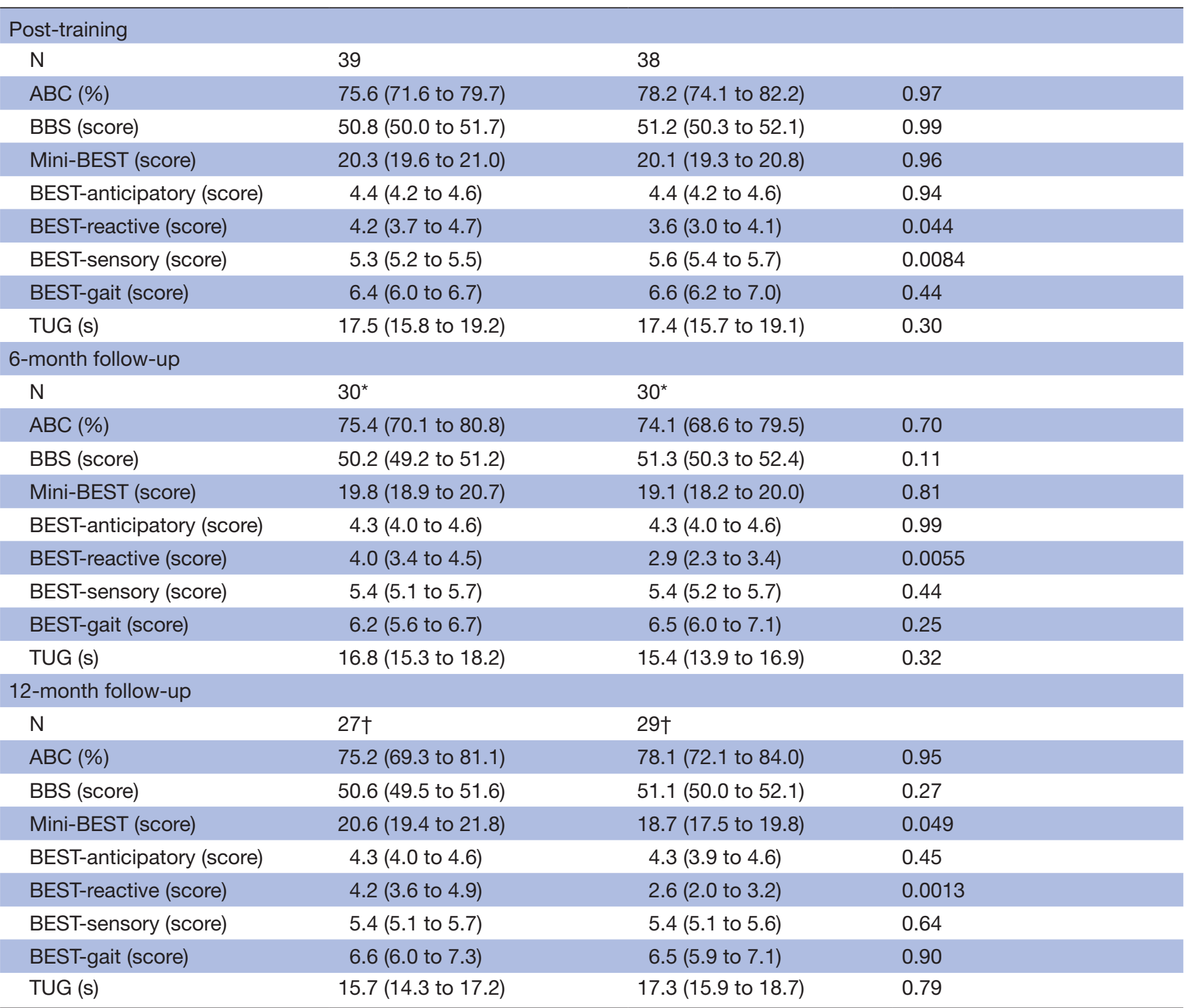

Values presented are least-square means with $95 \%$ confidence intervals in brackets. The $p$ value is for the ANCOVA comparing groups at each time point controlling for the baseline value.

${ }^{*} n=32$ PBTs and 31 controls for the ABC at 6 -month follow-up.

$\mathrm{tn}=31 \mathrm{PBT}$ and 31 controls for the ABC at 12-month follow-up.

ABC, activities specific balance confidence scale; ANCOVA, analysis of covariance; BBS, Berg balance scale; BEST, balance evaluation systems test; TUG, Timed Up \& Go.

the study). For all but this last event, medical attention was not necessary to treat adverse events. In the case of fatigue or joint/muscle pain, the intensity and/or duration of training was reduced until the issue resolved. Additionally, four falls that occurred during the training portion of the study were considered related to study procedures or interventions. In one case (control), the participant fell outside the hospital while on the way to a study appointment. The other three falls were reported by a single PBT participant who noted that he felt more confident and may have increased risk-taking behaviour, as a result of the intervention. Eight participants experienced serious adverse events unrelated to study procedures but that resulted in study withdrawal: prolonged hospitalisation (one PBT and one control participant); another stroke (two PBT and three control participants); death (one control participant); and cancer diagnosis (one control participant).

\section{DISCUSSION}

We hypothesised that PBT would reduce fall rates among individuals with stroke; this hypothesis was not supported. While the rate ratio comparing falls rates between the $\mathrm{PBT}$ and control groups was 0.85 , this was not statistically 
Table 4 Physical activity and social integration between groups

PBT Control P values

\begin{tabular}{|c|c|c|c|}
\hline \multicolumn{4}{|l|}{ Post-training } \\
\hline $\mathrm{N}$ & 39 & 38 & \\
\hline PASIPD (score) & 12.3 (10.0 to 14.6$)$ & 11.2 (8.8 to 13.6$)$ & 0.92 \\
\hline SIPSO (score) & 29.8 (28.1 to 31.4$)$ & 31.2 (29.5 to 32.9$)$ & 0.29 \\
\hline \multicolumn{4}{|l|}{ 2-month follow-up } \\
\hline $\mathrm{N}$ & 38 & 31 & \\
\hline PASIPD (score) & 8.6 (6.4 to 10.8$)$ & 9.5 (7.1 to 11.9$)$ & 0.51 \\
\hline SIPSO (score) & 29.7 (28.2 to 31.2) & 31.5 (29.8 to 33.21$)$ & 0.23 \\
\hline \multicolumn{4}{|l|}{ 4-month follow-up } \\
\hline $\mathrm{N}$ & 33 & 34 & \\
\hline PASIPD (score) & 9.2 (7.3 to 11.2 ) & 7.8 (5.9 to 9.8$)$ & 0.34 \\
\hline SIPSO (score) & 30.0 (28.2 to 31.9$)$ & 30.2 (28.4 to 32.0$)$ & 0.62 \\
\hline \multicolumn{4}{|l|}{ 6-month follow-up } \\
\hline $\mathrm{N}$ & 32 & $31^{*}$ & \\
\hline PASIPD (score) & $11.3(7.3$ to 15.3$)$ & $10.9(6.8$ to 15.0$)$ & 0.21 \\
\hline SIPSO (score) & 30.3 (29.0 to 31.6) & 32.6 (31.3 to 33.9 ) & 0.012 \\
\hline \multicolumn{4}{|l|}{ 8-month follow-up } \\
\hline $\mathrm{N}$ & 31 & 26 & \\
\hline PASIPD (score) & 7.0 (5.6 to 8.4 ) & 6.9 (5.4 to 8.5$)$ & 0.61 \\
\hline SIPSO (score) & 30.5 (29.3 to 31.7$)$ & 32.3 (31.0 to 33.6 ) & 0.037 \\
\hline \multicolumn{4}{|l|}{ 10-month follow-up } \\
\hline $\mathrm{N}$ & 32 & 32 & \\
\hline PASIPD (score) & 7.0 (5.5 to 8.5$)$ & 8.2 (6.7 to 9.7$)$ & 0.16 \\
\hline SIPSO (score) & 29.9 (28.4 to 31.3$)$ & 32.3 (30.9 to 33.8 ) & 0.031 \\
\hline \multicolumn{4}{|l|}{ 12-month follow-up } \\
\hline $\mathrm{N}$ & 31 & 31 & \\
\hline PASIPD (score) & $11.1(7.4$ to 14.8$)$ & $10.1(6.4$ to 13.9$)$ & 0.27 \\
\hline SIPSO (score) & 30.6 (29.1 to 32.0$)$ & 32.6 (31.1 to 34.0$)$ & 0.047 \\
\hline
\end{tabular}

Values presented are least square means with $95 \%$ confidence intervals in brackets. The $p$ value is for the ANCOVA comparing groups at each time point controlling for the baseline value.

${ }^{*} n=30$ control for the SIPSO.

ANCOVA, analysis of covariance; PASIPD, Physical Activity Scale for Individuals with Physical Disabilities; PBT, perturbation-based balance training; SIPSO, Subjective Index of Physical and Social Outcome.

significant. The pooled rate ratio estimating the effect of exercise on fall rates in community-dwelling older adults is $0.80,{ }^{4}$ which is similar to that observed in the current study. Our sample size was based on a rate ratio of 0.54 , which was estimated from a meta-analysis of $\mathrm{PBT}^{14}$ that included studies among older adults and individuals with Parkinson's disease. Another non-randomised study reported a fall rate ratio of 0.32 when comparing individuals with subacute stroke who completed PBT during inpatient rehabilitation with those who did not. ${ }^{20}$ The effect of PBT on fall rates in chronic stroke may be much lower than in other patient populations or individuals with subacute stroke, and therefore, the current study may not have had sufficient power to detect the true effect. Conversely, the between-group difference in fall rates was much greater when only individuals who completed at least $80 \%$ of initial training sessions and one booster session were included in the analysis. The booster sessions may have helped participants to retain the training benefits ${ }^{38} 39$ by providing participants with opportunity to practice reactive balance skills throughout the 12-month follow-up period.

Importantly, the control group also completed balance training; previous studies using similar exercise programmes found no effect of balance training on fall rates in people with chronic stroke when compared with a sham intervention ${ }^{7}$ or 'usual care'. ${ }^{40}$ Thus, we expect that control participants did not have reduced fall risk as a result of completing this programme. However, after the initial 6-week training period, both groups improved 
Table 5 Between-group comparison of fall circumstances

$\frac{\text { PBT }}{\text { (53 falls) }} \frac{\text { Control }}{\text { (64 falls) }}$ P values

Cause of fall

$\begin{array}{lccc}\text { Do not recall } & 8 & 6 & \\ \text { Slip } & 16(35.6) & 22(37.9) & 0.26 \\ \text { Trip } & 11(24.4) & 6(10.3) & \\ \text { Push/external force } & 1(2.2) & 3(5.2) & \\ \begin{array}{l}\text { Incorrect weight } \\ \text { transfer }^{53}\end{array} & 17(37.8) & 27(46.6) & \end{array}$

Posture at the time of the fall

\begin{tabular}{|lcll}
\hline Do not recall & 7 & 4 & \\
\hline Lying & $1(2.2)$ & $0(0)$ & 0.33 \\
\hline Sitting & $4(8.9)$ & $7(12.1)$ & \\
\hline Standing & $9(20.0)$ & $6(10.3)$ & \\
\hline Walking & $32(68.9)$ & $47(77.6)$ & \\
$\begin{array}{l}\text { Motor activity at the time of the fall } \\
\text { Do not recall }\end{array}$ & 7 & 4 & \\
\hline Not moving & $4(8.9)$ & $2(3.5)$ & 0.01 \\
\hline $\begin{array}{l}\text { Transferring } \\
\text { Turning/reaching/ } \\
\text { bending }\end{array}$ & $2(4.4)$ & $12(20.7)$ & \\
$\begin{array}{l}\text { Walking on level } \\
\text { surface }\end{array}$ & $18(37.8)$ & $20(34.5)$ & \\
$\begin{array}{l}\text { Walking on ramp/ } \\
\text { stairs/uneven } \\
\text { surface }\end{array}$ & $12(26.7)$ & $22(36.2)$ & \\
\hline
\end{tabular}

Cognitive activity at the time of the fall

\begin{tabular}{|c|c|c|c|}
\hline Do not recall & 10 & 9 & \\
\hline None & 34 (78.6) & $44(81.1)$ & 0.8 \\
\hline Distracted & $9(21.4)$ & $11(18.9)$ & \\
\hline \multicolumn{4}{|c|}{ Where did the fall occur } \\
\hline Outdoors & $19(35.8)$ & $22(34.4)$ & $>0.99$ \\
\hline Indoors & $34(64.2)$ & $42(65.6)$ & \\
\hline \multicolumn{4}{|c|}{ Using an assistive device } \\
\hline Do not recall & 7 & 5 & \\
\hline Never use one & 11 & 23 & \\
\hline No & $16(45.7)$ & $24(66.7)$ & 0.096 \\
\hline Yes & 19 (54.3) & $12(33.3)$ & \\
\hline \multicolumn{4}{|c|}{ Holding onto a handrail } \\
\hline Do not recall & 7 & 6 & \\
\hline No & $41(89.1)$ & $48(82.8)$ & 0.41 \\
\hline Yes & $5(10.9)$ & $10(17.2)$ & \\
\hline \multicolumn{4}{|l|}{ Anything in hands } \\
\hline Do not recall & 9 & 6 & \\
\hline No & 34 (77.3) & $32(55.2)$ & 0.023 \\
\hline $\begin{array}{l}\text { Yes (one or both } \\
\text { hands) }\end{array}$ & $10(22.7)$ & $26(44.8)$ & \\
\hline
\end{tabular}

Continued
Table 5 Continued

$\frac{\text { PBT }}{\text { (53 falls) }} \frac{\text { Control }}{\text { (64 falls) }}$ P values

Action to try to prevent the fall

\begin{tabular}{|c|c|c|c|}
\hline Do not recall & 9 & 18 & \\
\hline None & $27(61.4)$ & $28(60.9)$ & 0.18 \\
\hline Grasp & $8(18.2)$ & $14(30.4)$ & \\
\hline Step or step+grasp & $9(20.5)$ & $4(8.7)$ & \\
\hline \multicolumn{4}{|c|}{ Length of lie on floor or ground } \\
\hline Do not recall & 7 & 4 & \\
\hline $\begin{array}{l}\text { A few minutes or } \\
\text { less }\end{array}$ & $39(84.8)$ & $57(95.0)$ & 0.098 \\
\hline $\begin{array}{l}\text { More than a few } \\
\text { minutes but less } \\
\text { than an hour }\end{array}$ & $7(15.2)$ & $3(5.0)$ & \\
\hline \multicolumn{4}{|c|}{ Assistance required to get up from fall } \\
\hline Do not recall & 7 & 4 & \\
\hline No & $24(52.2)$ & 44 (73.3) & 0.04 \\
\hline Yes & $22(47.8)$ & $16(26.7)$ & \\
\hline \multicolumn{4}{|l|}{ Injuries } \\
\hline Do not recall & 7 & 5 & \\
\hline None & $28(60.9)$ & $39(66.1)$ & $0.68^{*}$ \\
\hline Cuts or bruises & $17(37.0)$ & $19(32.2)$ & \\
\hline $\begin{array}{l}\text { Joint sprain or } \\
\text { dislocation }\end{array}$ & $1(2.2)$ & $1(1.7)$ & \\
\hline \multicolumn{4}{|c|}{ Medical assistance required after fall } \\
\hline Do not recall & 7 & 5 & \\
\hline No injuries & 30 & 42 & \\
\hline $\begin{array}{l}\text { Injured but did not } \\
\text { seek treatment }\end{array}$ & $16(100)$ & $14(82.4)$ & $0.23+$ \\
\hline $\begin{array}{l}\text { Saw other } \\
\text { healthcare } \\
\text { professional }\end{array}$ & $0(0)$ & $1(5.9)$ & \\
\hline $\begin{array}{l}\text { Treated in hospital } \\
\text { emergency room }\end{array}$ & $0(0)$ & $2(11.8)$ & \\
\hline
\end{tabular}

Values are the number of falls in each category, with the percentage of falls in parentheses. The percentage was calculated from the total number of falls for which information was available (ie, 'do not recall' responses were excluded from the denominator). Percentages might not sum to 100 due to rounding error. The $p$ value is for Fisher's exact test comparing the two groups, excluding 'do not recall' responses from analysis.

*Analysis compared injury versus no injury.

†Analysis compared sought treatment versus did not seek treatment.

PBT, perturbation-based balance training.

balance confidence (ABC), anticipatory balance control (BBS and mini-BEST anticipatory subscale sore)and mobility (mini-BEST gait subscale score), with no significant difference between groups on these measures posttraining. It is possible that improved balance and mobility led to reduced fall risk in the both groups compared with their pretraining fall risk. Furthermore, it seems that PBT 
leads to similar improvements in anticipatory balance and mobility as a traditional balance training programme that is primarily focused on improving anticipatory balance control.

Consistent with specificity of training, the PBT group improved reactive balance control (reactive subscale of the mini-BEST), but the control group did not ${ }^{41}$; these improvements were retained at 6 months and 12 months. This finding agrees with those of Bhatt $e t a l^{39}$, who found that resistance to falling following a slip was retained up to 6 months after a single PBT session. The mean between-group difference in the reactive subscale of the mini-BEST ranged from 0.6 (post-training) to 1.6 points (12-month follow-up). We are unaware of any study reporting minimal clinically important differences for the mini-BEST subscales; however, these between group differences represent $10 \%-27 \%$ of the maximum score for this subscale (six points), and therefore, we interpret these differences as clinically meaningful. Despite these retained improvements in reactive balance control, PBT participants did not have a significantly reduced fall risk than control participants. Falls occur when there is a loss of balance and subsequent failure to recover. ${ }^{42}$ Improved reactive balance control following PBT should help to prevent falls by improving the ability to recover from a loss of balance. Loss of balance can occur due to an external force or failure of anticipatory balance control. Thus, it is possible that effective fall prevention poststroke requires sustained improvements in both anticipatory and reactive balance control; home exercise may help participants to retain improvements in anticipatory balance control. ${ }^{40}$

Contrary to our hypothesis, control participants reported greater social integration 6-12 months posttraining than the PBT group. Individual-item SIPSO scores suggest that this finding was primarily driven by control participants reporting increased independence in moving around their local neighbourhoods. The control training programme included walking practice during every session, whereas the PBT programme only included short bouts of walking in later sessions. This walking practice may have increased control participants' confidence with community mobility. While increased social integration at 6-12 months was not associated with improved physical function, it is likely that the tests used in the current study do not correlate highly with community mobility. ${ }^{43}$ Training-related improvements in balance and mobility in both groups, and increased self-reported participation in the control group, were not associated with increased physical activity post-training. While impaired balance and mobility poststroke may be a barrier to physical activity, ${ }^{44}$ improved balance and mobility alone is not sufficient to increase activity. ${ }^{75} \mathrm{It}$ is likely that an intervention that combines behaviour change techniques with physical exercise is required to increase long-term participation in physical activity. ${ }^{46}$

Examining fall characteristics can provide further insight into intervention effects on falls. ${ }^{20}$ Individuals with stroke seem to be reliant on upper extremity reactions to prevent falls in daily life. ${ }^{29}$ In the current study, participants had something in their hands at the time of the fall for more control group falls than PBT falls, which may have prevented these individuals from using an upper extremity reaction to prevent the fall. ${ }^{47}$ Conversely, training, with a specific focus on reactive stepping, may have made PBT participants less reliant on upper extremity reactions to prevent falls. In agreement with a previous study, ${ }^{20}$ control participants were more likely than PBT participants to fall during transfers; this finding may support the idea that PBT helps to prevent falls in routine situations but not falls in more challenging situations. Participants required assistance to get up from the ground after more PBT group than control group falls; this finding could suggest that those PBT participants who fell were more impaired than PBT participants who did not fall or than those in the control group who fell.

\section{Limitations}

The primary outcome (falls in daily life) was obtained via self-report. While the method of prospective falls reporting used in the current study is the best available, ${ }^{48}$ falls may have been under-reported. The cohort was, on average, relatively high functioning (eg, median BBS score $\sim 50 / 56$ ) but had a wide range of physical function (minimum scores for CMSA leg: 3, CMSA foot: 2, BBS: 23, mini-BEST: 5; maximum NIH-SS score: 13; and highest TUG time: $119 \mathrm{~s}$ ). This study's findings apply to community-dwelling individuals with chronic stroke who can stand independently for at least $30 \mathrm{~s}$. Group allocation blinding was violated for nine participants. Balance measures for these participants were rescored by a truly blinded research assistant; however, knowledge of group allocation may have subconsciously influenced how other data were collected for these participants.

PASIPD scores were higher at the time points when the questionnaire was administered in person compared with over the telephone. Physical activity questionnaires, including the PASIPD, ${ }^{34}$ are often designed to have several methods of administration (eg, self-administered via in-person or telephone interview) ${ }^{49}$ and investigators seem to treat administration methods as equivalent. ${ }^{50} \mathrm{We}$ are not aware of any study that directly compared scores from the PASIPD or any other physical activity questionnaire when administered using different methods. It is possible that scores are higher when administered in-person versus over the telephone as participants' desire for social acceptance was higher when they interacted directly with the research assistant. Alternatively, in-person administration may have led to more accurate scores than telephone administration within this population, who may have subtle cognitive communication deficits, as the research assistant and participant could avail of non-verbal communication to facilitate completing the questionnaire. However, SIPSO scores did not differ between telephone versus in-person administration. Finally, participants in the current study may have truly been more active in the week prior to the in-person 
interview compared with the telephone interview to prepare for the tests of physical function. Future studies should investigate the potential influence of administration methods on physical activity questionnaire scores.

\section{Clinical implications}

While this study found that PBT did not reduce fall rates among the entire cohort, PBT participants improved on measures of balance and mobility and retained the improvements in reactive balance control up to 12 months post-training. Combined with results of previous studies reporting reduced fall rates following PBT among individuals with subacute stroke, ${ }^{20}$ chronic stroke with a history of falling ${ }^{51}$ and without stroke ${ }^{14}$ and showing that PBT is the only intervention with capacity to improve reactive balance control, ${ }^{41}{ }^{52}$ these results suggest that PBT may be a useful addition to existing balance training poststroke. The PBT programme developed for this study used existing resources available in many clinical settings and, therefore, could be relatively easily implemented in clinical practice. Joint pain was the most common adverse event related to PBT, which appeared to be most prevalent among those with lower-extremity arthritis; these participants were able to complete training with modifications to avoid exacerbating pain (eg, temporarily reducing perturbation intensity). Therefore, modifications to PBT may be required for those with lower extremity arthritis. Regular 'booster' PBT training sessions may be necessary to prevent falls long term.

\section{Author affiliations}

${ }^{1}$ Toronto Rehabilitation Institute, University Health Network, Toronto, Ontario, Canada ${ }^{2}$ Evaluative Clinical Sciences, Hurvitz Brain Sciences Research Program, Sunnybrook Research Institute, Toronto, Ontario, Canada

${ }^{3}$ Department of Physical Therapy, University of Toronto, Toronto, Ontario, Canada ${ }^{4}$ School of Rehabilitation Therapy, Queen's University, Kingston, Ontario, Canada ${ }^{5}$ Schulich School of Education - School of Physical and Health Education, Nipissing University, North Bay, Ontario, Canada

\section{Twitter@simbl_to @avrilmansfield @depaulvg @brooksdi @elizinness @} georgemochizuki

Acknowledgements We would like to thank the members of the Data Safety and Monitoring Committee (Kathryn Sibley, Susan Marzolini, Parvin Eftekhar and Irene Antunes) who monitored the trial. We also acknowledge Rabea Aryan and Ellen Cohen for assistance with administering the intervention, Jyoti Mann and Andrew Huntley for assistance with data processing and Cynthia Campos for assistance with creating the training manual.

Contributors AM conceived of the study, is the grant holder, performed statistical analysis and drafted the manuscript. AM, VGD, AS-I, DB, ELI and GM developed the study protocol. AM and GM led implementation of the study at each site. AM, VGD and ELI developed the intervention. AA, AC and AS-I collected data. CJD and SK delivered the interventions. All authors approved the final manuscript.

Funding This study was supported by the Canadian Institutes of Health Research (MOP 133577). The authors also acknowledge the support of the Toronto Rehabilitation Institute; equipment and space have been funded with grants from the Canada Foundation for Innovation, Ontario Innovation Trust and the Ministry of Research and Innovation. AM holds a New Investigator Award from the Canadian Institutes of Health Research (MSH 141983). DB holds a Canada Research Chair.

Disclaimer These funding sources had no role in the design of this study and will not have any role during its execution, analyses, interpretation of the data or decision to submit results.

Competing interests None declared.
Patient consent Obtained.

Ethics approval University Health Network and Sunnybrook Health Sciences Centre.

Provenance and peer review Not commissioned; externally peer reviewed.

Data sharing statement Due to research ethics and privacy restrictions, raw data for this study are currently not available publicly.

Open access This is an open access article distributed in accordance with the Creative Commons Attribution Non Commercial (CC BY-NC 4.0) license, which permits others to distribute, remix, adapt, build upon this work non-commercially, and license their derivative works on different terms, provided the original work is properly cited, appropriate credit is given, any changes made indicated, and the use is non-commercial. See: http://creativecommons.org/licenses/by-nc/4.0/.

\section{REFERENCES}

1. Batchelor FA, Mackintosh SF, Said CM, et al. Falls after stroke. Int J Stroke 2012;7:482-90.

2. Andersson AG, Kamwendo K, Appelros P. Fear of falling in stroke patients: relationship with previous falls and functional characteristics. Int J Rehabil Res 2008;31:261-4.

3. Schmid AA, Van Puymbroeck M, Altenburger PA, et al. Balance and balance self-efficacy are associated with activity and participation after stroke: a cross-sectional study in people with chronic stroke. Arch Phys Med Rehabil 2012;93:1101-7.

4. Sherrington $\mathrm{C}$, Michaleff ZA, Fairhall N, et al. Exercise to prevent falls in older adults: an updated systematic review and meta-analysis. $\mathrm{Br}$ J Sports Med 2017;51 17501758.

5. Verheyden GS, Weerdesteyn V, Pickering RM, et al. Interventions for preventing falls in people after stroke. Cochrane Database Syst Rev 2013;31:CD008728.

6. Batchelor F, Hill K, Mackintosh S, et al. What works in falls prevention after stroke?: a systematic review and meta-analysis. Stroke 2010;41:1715-22.

7. Dean CM, Rissel C, Sherrington C, et al. Exercise to enhance mobility and prevent falls after stroke: the community stroke club randomized trial. Neurorehabil Neural Repair 2012;26:1046-57.

8. Gardner MM, Buchner DM, Robertson MC, et al. Practical implementation of an exercise-based falls prevention programme. Age Ageing 2001;30:77-83.

9. Dean CM, Richards CL, Malouin F. Task-related circuit training improves performance of locomotor tasks in chronic stroke: a randomized, controlled pilot trial. Arch Phys Med Rehabil 2000;81:409-17.

10. Eng JJ, Chu KS, Kim CM, et al. A community-based group exercise program for persons with chronic stroke. Med Sci Sports Exerc 2003;35:1271-8.

11. Salbach NM, Mayo NE, Wood-Dauphinee S, et al. A task-orientated intervention enhances walking distance and speed in the first year post stroke: a randomized controlled trial. Clin Rehabil 2004;18:509-19.

12. Maki BE, Mcllroy WE. The role of limb movements in maintaining upright stance: the "change-in-support" strategy. Phys Ther 1997;77:488-507.

13. Gerards MHG, McCrum C, Mansfield A, et al. Perturbation-based balance training for falls reduction among older adults: current evidence and implications for clinical practice. Geriatr Gerontol Int 2017;17:2294-303.

14. Mansfield A, Wong JS, Bryce J, et al. Does perturbation-based balance training prevent falls? Systematic review and meta-analysis of preliminary randomized controlled trials. Phys Ther 2015;95:700-9.

15. Mansfield A, Inness EL, Lakhani B, et al. Determinants of limb preference for initiating compensatory stepping poststroke. Arch Phys Med Rehabil 2012;93:1179-84.

16. de Kam D, Roelofs JMB, Bruijnes A, et al. The next step in understanding impaired reactive balance control in people with stroke: the role of defective early automatic postural responses. Neurorehabil Neural Repair 2017;31:708-16.

17. Mansfield A, Wong JS, Mcllroy WE, et al. Do measures of reactive balance control predict falls in people with stroke returning to the community? Physiotherapy 2015;101:373-80.

18. Mansfield A, Inness EL, Wong JS, et al. Is impaired control of reactive stepping related to falls during inpatient stroke rehabilitation? Neurorehabil Neural Repair 2013;27:526-33.

19. Mansfield A, Inness EL, Komar J, et al. Training rapid stepping responses in an individual with stroke. Phys Ther 2011;91:958-69. 
20. Mansfield A, Schinkel-Ivy A, Danells CJ, et al. Does perturbation training prevent falls after discharge from stroke rehabilitation? a prospective cohort study with historical control. J Stroke Cerebrovasc Dis 2017;26:2174-80.

21. Mansfield A, Aqui A, Centen A, et al. Perturbation training to promote safe independent mobility post-stroke: study protocol for a randomized controlled trial. BMC Neurol 2015;15:87.

22. Schulz KF, Altman DG, Consort MD. statement: updated guidelines for reporting parallel group randomised trials. $\mathrm{Br}$ Med J 2010;2010:c332.

23. Hoffman TC, Glasziou PP, Milne R, et al. Better reporting of interventions: template for intervention desription and replication (TIDieR) checklist and guide. Br Med J 2014;345:1687.

24. Thomas S, Reading J, Shephard RI. Revision of the Physical Activity Readiness Questionnaire (PAR-Q). Can J Sport Sci 1992;174:338-45.

25. French E, Reinikka K, MacLeod A. Community-based exercise for people living with stroke. http://www.tbrhsc.net/clinical_partners/ regional_stroke_program/video_resources/community_based_ exercise.asp (accessed 1 Aug 2013).

26. Shumway-Cook A, Woollacott MH. Motor control: translating research into clinical practice. 3rd edn. Philadelphia: Lippincott Williams \& Wilkins, 2007.

27. Goldstein LB, Bertels C, Davis JN. Interrater reliability of the NIH stroke scale. Arch Neurol 1989;46:660-2.

28. Gowland $\mathrm{C}$, Stratford $\mathrm{P}$, Ward M, et al. Measuring physical impairment and disability with the chedoke-mcmaster stroke assessment. Stroke 1993;24:58-63.

29. Hyndman D, Ashburn A, Stack E. Fall events among people with stroke living in the community: circumstances of falls and characteristics of fallers. Arch Phys Med Rehabil 2002;83:165-70.

30. Berg K, Wood-Dauphinée S, Williams JI, Gayton D. Measuring balance in the elderly: preliminary development of an instrument. Physiotherapy Canada 1989;41:304-11.

31. Franchignoni F, Horak F, Godi M, et al. Using psychometric techniques to improve the balance evaluation systems test: the miniBEStest. J Rehabil Med 2010;42:323-31.

32. Podsiadlo D, Richardson S. The timed "Up \& Go": a test of basic functional mobility for frail elderly persons. J Am Geriatr Soc 1991;39:142-8.

33. Powell LE, Myers AM. The Activities-specific Balance Confidence (ABC) scale. J Gerontol A Biol Sci Med Sci 1995;50A:M28-34.

34. Washburn RA, Zhu W, McAuley E, et al. The physical activity scale for individuals with physical disabilities: development and evaluation. Arch Phys Med Rehabil 2002;83:193-200.

35. Trigg R, Wood VA. The Subjective Index of Physical and Social Outcome (SIPSO): a new measure for use with stroke patients. Clin Rehabil 2000;14:288-99.

36. Tang Y. Sample size estimation for negative binomial regression comparing rates of recurrent events with unequal follow-up time. $J$ Biopharm Stat 2015;25:1100-13.

37. Conover WJ, Iman RL. Analysis of covariance using the rank transformation. Biometrics 1982;38:715-24.

38. van Duijnhoven HJ, De Kam D, Hellebrand W, et al. Development and process evaluation of a 5-week exercise program to prevent falls in people after stroke: the falls program. Stroke Res Treat 2012;2012:1-7.

39. Bhatt T, Yang F, Pai YC. Learning to resist gait-slip falls: long-term retention in community-dwelling older adults. Arch Phys Med Rehabil 2012;93:557-64.

40. Batchelor FA, Hill KD, Mackintosh SF, et al. Effects of a multifactorial falls prevention program for people with stroke returning home after rehabilitation: a randomized controlled trial. Arch Phys Med Rehabil 2012;93:1648-55.

41. Mansfield A, Peters AL, Liu BA, et al. Effect of a perturbation-based balance training program on compensatory stepping and grasping reactions in older adults: a randomized controlled trial. Phys Ther 2010;90:476-91.

42. Maki BE, Mcllroy WE. Postural control in the older adult. Clin Geriatr Med 1996;12:635-58.

43. Robinson CA, Shumway-Cook A, Matsuda PN, et al. Understanding physical factors associated with participation in community ambulation following stroke. Disabil Rehabil 2011;33:1033-42.

44. Nicholson S, Sniehotta FF, van Wijck F, et al. A systematic review of perceived barriers and motivators to physical activity after stroke. Int J Stroke 2013;8:357-64.

45. Mudge S, Barber PA, Stott NS. Circuit-based rehabilitation improves gait endurance but not usual walking activity in chronic stroke: a randomized controlled trial. Arch Phys Med Rehabil 2009;90:1989-96.

46. Morris JH, Macgillivray S, McFarlane S. Interventions to promote long-term participation in physical activity after stroke: a systematic review of the literature. Arch Phys Med Rehabil 2014;95:956-67.

47. Bateni H, Zecevic A, Mcllroy WE, et al. Resolving conflicts in task demands during balance recovery: does holding an object inhibit compensatory grasping? Exp Brain Res 2004;157:49-58.

48. Lamb SE, Jørstad-Stein EC, Hauer K, et al. Development of a common outcome data set for fall injury prevention trials: the Prevention of Falls Network Europe consensus. J Am Geriatr Soc 2005;53:1618-22.

49. Strath SJ, Kaminsky LA, Ainsworth BE, et al. Guide to the assessment of physical activity: clinical and research applications: a scientific statement from the American Heart Association. Circulation 2013;128:2259-79.

50. Craig CL, Marshall AL, Sjöström M, et al. International physical activity questionnaire: 12 -country reliability and validity. $\mathrm{Med} \mathrm{Sci}$ Sports Exerc 2003;35:1381-95

51. Marigold DS, Eng JJ, Dawson AS, et al. Exercise leads to faster postural reflexes, improved balance and mobility, and fewer falls in older persons with chronic stroke. J Am Geriatr Soc 2005;53:416-23

52. Smith GV, Forrester LW, Silver KHC, et al. Effects of treadmill training on translational balance perturbation responses in chronic hemiparetic stroke patients. J Stroke Cerebrovasc Dis 2000;9:238-45.

53. Robinovitch SN, Feldman F, Yang Y, et al. Video capture of the circumstances of falls in elderly people residing in long-term care: an observational study. Lancet 2013;381:47-54. 